\title{
MAPEAMENTO DE METODOLOGIAS DE AVALIAÇÃO DE PROJETOS SOCIAIS
}

\section{Patrícia Peres Rodrigues (D1, Bruna Angela Branchi (D), Cibele Roberta Sugahara iD 3}

Resumo: As organizações sociais desenvolvem programas que contribuem para o bem-estar social e o atendimento de lacunas do setor público, ao fomentar o exercício da cidadania de forma direta e autônoma. O conhecimento dos impactos destes programas é fundamental na comunicação com os diferentes stakeholders. Tendo em vista isso, o artigo objetiva mapear as metodologias de avaliação dos impactos de programas sociais a partir de uma pesquisa bibliográfica descritiva-explicativa. No desenvolvimento do trabalho foram consultadas as bases SciELO, Scopus e BDTD no sentido de investigar a produção científica sobre o tema avaliação de programas sociais ao longo dos últimos dez anos. Constatou-se que poucos trabalhos acadêmicos foram desenvolvidos e publicados, assinalando a necessidade de fomentar a cultura da avaliação, especialmente para colaborar com os gestores das organizações durante o processo avaliativo dos impactos dos programas desenvolvidos.

Palavras-chave: Avaliação de impacto; Metodologias de avaliação; Programas sociais.

\section{MAPPING OF SOCIAL PROJECT EVALUATION METHODOLOGIES}

Abstract: Social organizations develop programs that contribute to social wellbeing and fill gaps in the public sector by fostering the exercise of citizenship, directly and autonomously. Knowledge of the impacts of these programs is essential when communicating with different stakeholders. Therefore, this paper aims to map the methodologies used for assessing social programs impacts through a descriptive-explanatory bibliographic research. In developing the study, the SciELO, Scopus, and BDTD databases were consulted in the sense to investigate the scientific production on social program evaluation over the past ten years. It was found that few academic works were developed and published. This result highlights the need to foster the evaluation culture, especially to collaborate with the managers of organizations when evaluating the impacts of the programs developed.

Keywords: Impact assessment; Evaluation methodologies; Social programs.

\section{Introdução}

\footnotetext{
${ }^{1}$ Mestra em Sustentabilidade pela Pontifícia Universidade Católica de Campinas (PUC/Campinas). Supervisora de Marketing e Sustentabilidade da BYD Brasil Ltda. E-mail: ptcperes@hotmail.com. ${ }^{2}$ Doutorada em Economia Política pela Universitá degli Studi de Pavia (Itália). Professora e Pesquisadora do Programa de Pós-Graduação em Sustentabilidade da PUC/Campinas. E-mail: bruna.branchi@puc-campinas.edu.br.

${ }^{3}$ Doutorada em Ciência da Informação pela Universidade de São Paulo (USP). Professora e Pesquisadora do Programa de Pós-Graduação em Sustentabilidade da PUC/Campinas. E-mail: cibelesu@puc-campinas.edu.br.
} 
A forma como as organizações realizam a avaliação de programas sociais pode fragilizar a credibilidade das Organizações da Sociedade Civil (OSCs) perante as instituições e investidores interessados em apoiar 0 empreendimento.

A avaliação de programas sociais apresenta-se como um desafio para os gestores das OSCs. Segundo Assumpção e Campos (2011), quando a avaliação é aplicada restringe-se ao controle dos investimentos financeiros ou para fins de relatório de atividades desenvolvidas, nem sempre conferindo valor ou mérito à iniciativa.

Quanto ao acesso dos gestores à informação sobre as metodologias de avaliação de programas sociais, faltam estudos acadêmicos que demonstrem o acompanhamento e análise de programas sociais e que possibilitem que as instituições demonstrem o retorno social das intervenções. Ocorre também a ausência de materiais que auxiliem, de forma didática, os atores sociais a avaliarem as intervenções além dos aspectos da economia, eficiência e eficácia (ASSUMPÇÃO e CAMPOS, 2011).

Nesta pesquisa, a opção de mapear as metodologias de avaliação de programas sociais, justifica-se pela contribuição dessas ferramentas na construção de cenários fidedignos que descrevam os efeitos das ações dos programas sociais e possibilitem o seu aperfeiçoamento. Além disso, mapear a evolução dos programas possibilita gerar credibilidade perante aos stakeholders e à sociedade (ROCHE, 2000).

Diante disso, depreende-se que os gestores das OSCs precisam de estímulo e auxílio na realização da avaliação dos programas sociais que estas instituições realizam. Gasparini e Furtado (2014), em um trabalho de revisão sistemática sobre a avaliação de Programas e Serviços Sociais no Brasil, apontam que a avaliação de projetos sociais por agentes externos, ou seja, que não fazem parte da instituição, constitui a maioria dos processos avaliativos. Os autores analisam que este fato pode ser um indicativo de que as avaliações de impacto aconteçam esporadicamente, não interferindo de maneira substancial no processo de tomada de decisões.

Sendo assim, entende-se que a forma como as organizações realizam a avaliação de programas sociais pode fragilizar a credibilidade das OSCs, que têm dificuldade em relatar seus avanços. Bengo et al. (2016) pontuam que a falta de indicadores e métricas de avaliação de impacto confiáveis limitam a capacidade das organizações sociais de competir no cenário socioeconômico por três razões: 1) diminui a capacidade das organizações sociais de atraírem recursos por meio de investimentos e doações, uma vez que os potenciais investidores/doadores não possuem instrumentos confiáveis e reconhecidos para avaliar o potencial e os resultados de seus investimentos e compará-los com outras opções de investimento; 2) limita a possibilidade de obterem o apoio dos formuladores de políticas; 3) enfraquece a capacidade dos próprios gerentes de planejar e controlar suas atividades operacionais e de se comunicar com a ampla gama de stakeholders.

Murad (2017) enfatiza que a captação de recursos é essencial para a satisfação das necessidades de recursos financeiros e humanos das OSCs, mantendo a capacidade de atuação da organização. Entretanto, adverte que 
devido ao grande número de OCS, a captação de recursos torna-se uma atividade árdua e que demanda grande dedicação dos gestores, pois os investidores precisam conhecer os resultados e impactos alcançados pelas OSCs antes de decidirem pelo investimento.

O Instituto para o Desenvolvimento do Investimento Social (IDIS), com a colaboração da Fundación Avina, a empresa de pesquisa Plano CDE e a Yunus Negócios Sociais, publicou uma pesquisa sobre a avaliação de impacto e as metodologias mais utilizadas em programas sociais no Brasil, intitulada "Avaliação de impacto: Metodologias e reflexões". O resultado evidenciou que, apesar de ser classificada como importante, a avaliação de programas sociais ainda é pouco conhecida e aplicada no país (FABIANI et al., 2018).

No estudo, Fabiani et al. (2018) apontam que as diferentes metodologias de medição de impacto são ainda desconhecidas entre os respondentes da pesquisa, portanto a maioria dos participantes não conseguiu opinar sobre elas, o que demonstra pouco conhecimento sobre o tema. Ao serem questionados sobre o interesse em participar de um curso de curta duração sobre metodologias de avaliação de impacto, muitos escolheram conhecer mais sobre as metodologias Social Return on Investment (SROI) e Teoria da Mudança, que é considerada uma "ferramenta a serviço da Avaliação de Impacto, e não uma metodologia de avaliação em si" (FABIANI et al., 2018, p. 6).

Endossando este raciocínio Cabral (2011) justifica que a avaliação de programas sociais e suas técnicas são pouco divulgadas às organizações sociais devido à falta de profissionalização destas empresas, por não haver ainda uma cultura sistematizada e difundida sobre o tema e pela ausência de uma metodologia própria para as ações sociais, sem a utilização de técnicas e premissas da área pública ou privada.

De maneira igualmente importante, Ribeiro (2017) afirma que é necessário progredir na execução da avaliação de impacto de programas sociais, pois considera que esta é uma tendência que tem relação com o cumprimento dos 17 Objetivos do Desenvolvimento Sustentável (ODS). A avaliação de impacto de programas sociais apresenta-se como um importante movimento para monitorar as ações direcionadas a atingir os ODS, que visam erradicar a pobreza, proteger o planeta e garantir a prosperidade para todos.

Com base nestas afirmações, parece haver a necessidade de estudos que denotem como a avaliação e a mensuração de impacto social no contexto das Organizações da Sociedade Civil é uma forma de alavancar as iniciativas orientadas para solução de problemas sociais.

Desse modo, esse trabalho tem por objetivo mapear as metodologias de avaliação utilizadas em projetos sociais. A construção dessa discussão é fundamentada em um levantamento bibliográfico conforme apresentado na próxima seção.

\section{Metodologia}

A pesquisa é de natureza aplicada e se ancora na abordagem qualiquantitativa. Segundo Richardson (2017, p.70), a pesquisa de métodos mistos 
tem "uma abordagem da investigação que combina ou associa as abordagens qualitativa e quantitativa".

A pesquisa caracteriza-se como bibliográfica descritiva-explicativa. Esta, por sua vez, busca retratar as características de determinada população ou fenômeno ou estabelecer relações sobre variáveis. A pesquisa explicativa tem como objetivo mapear os fatores que contribuem para a ocorrência de um fenômeno, aprofundando o conhecimento da realidade (GIL, 2008).

Para tanto, as fontes de dados utilizadas na pesquisa foram as bases de dados Scientific Electronic Library Online (SciELO), SciVerse Scopus e a Biblioteca Digital Brasileira de Teses e Dissertações (BDTD). Em cada uma dessas bases foram utilizados os seguintes descritores: "Avaliação de projetos sociais"; "Avaliação de programas sociais", "Metodologias de avaliação de impacto", "Social projects evaluation", "Social programs evaluation" e "Impact evaluation methodologies". O Quadro 1 apresenta a quantidade de trabalhos encontrados em cada base de dados por palavra-chave.

\section{Quadro 1 - Quantidade de trabalhos encontrados por palavra-chave e por base de dados.}

\begin{tabular}{|c|c|c|c|c|c|}
\hline Palavras-chave & \multirow{2}{*}{ SciELO } & Scopus & \multicolumn{2}{|c|}{$\begin{array}{c}\text { Biblioteca Digital de } \\
\text { Teses e Dissertações }\end{array}$} & $\begin{array}{c}\text { Total por } \\
\text { palavra- } \\
\text { chave }\end{array}$ \\
\cline { 4 - 6 } & Dissertações & Teses & $\mathbf{1 3}$ \\
\hline $\begin{array}{c}\text { Avaliação de projetos } \\
\text { Sociais }\end{array}$ & 5 & 3 & 5 & 0 & $\mathbf{1 8}$ \\
\hline $\begin{array}{c}\text { Avaliação de programas } \\
\text { sociais }\end{array}$ & 4 & 1 & 3 & 10 & $\mathbf{3}$ \\
\hline $\begin{array}{c}\text { Metodologias de } \\
\text { avaliação de impacto }\end{array}$ & 1 & 0 & 0 & 2 & $\mathbf{3}$ \\
\hline Social projects evaluation & 0 & 1 & 2 & - & $\mathbf{8}$ \\
\hline $\begin{array}{c}\text { Social programs } \\
\text { evaluation }\end{array}$ & 0 & 7 & & - & $\mathbf{1 3}$ \\
\hline $\begin{array}{c}\text { Impact evaluation } \\
\text { Methodologies }\end{array}$ & 1 & 11 & 1 & $\mathbf{1 3}$ & $\mathbf{5 8}$ \\
\hline $\begin{array}{c}\text { Total por base de } \\
\text { dados }\end{array}$ & $\mathbf{1 1}$ & $\mathbf{2 3}$ & $\mathbf{1 1}$ & \\
\hline
\end{tabular}

Fonte: Elaborado pelas autoras.

Com a pesquisa foram encontrados 58 trabalhos, sendo 34 artigos e 24 teses e dissertações. Tendo como critério não considerar trabalhos iguais, temse ao todo 41trabalhos distintos, sendo, 25 artigos e 16 teses e dissertações.

Considerando o grande número de OSCs no Brasil comparado com a produção científica limitada sobre o tema da avaliação de impacto de programas sociais nos últimos anos, este resultado pode indicar a falta de conhecimento dos gestores a respeito das metodologias de avaliação existentes (JANNUZZI, 2011).

O Quadro 2 permite observar que quinze artigos foram publicados em periódicos internacionais. Em geral, não há revistas repetidas, sendo duas exceções: Revista de Administração Pública e Meta:Análise. Cada uma delas publicou dois artigos. Além disso os dois artigos publicados na Revista de Administração Pública são os com o maior número de citações. 


\section{Quadro 2 - Quantidade de citações dos artigos encontrados na pesquisa.}

\begin{tabular}{|c|c|c|}
\hline Citações & Título, autor e ano & Periódico \\
\hline 30 & $\begin{array}{l}\text { Assumpção e Campos (2011). Avaliação de projetos sociais } \\
\text { em ONGs da Grande Florianópolis: um estudo sobre modelos } \\
\text { relacionados ao foco de atuação. }\end{array}$ & $\begin{array}{l}\text { Revista de } \\
\text { Administração } \\
\text { Pública }\end{array}$ \\
\hline 23 & $\begin{array}{l}\text { Cabral (2011). Valores e espaço público: referenciais e } \\
\text { instrumentos para a avaliação de projetos sociais. }\end{array}$ & $\begin{array}{l}\text { Revista de } \\
\text { Administração } \\
\text { Pública }\end{array}$ \\
\hline 22 & $\begin{array}{l}\text { Lillis (2012). Systematically evaluating the effectiveness of } \\
\text { quality assurance programmes in leading to improvements in } \\
\text { institutional performance. }\end{array}$ & $\begin{array}{l}\text { Quality in Higher } \\
\quad \text { Education }\end{array}$ \\
\hline 21 & $\begin{array}{l}\text { Ahmadia et al., (2015). Integrating impact evaluation in the } \\
\text { design and implementation of monitoring marine protected } \\
\text { areas. }\end{array}$ & $\begin{array}{l}\text { Philosophical } \\
\text { Transactions of the } \\
\text { Royal Society B: } \\
\text { Biological Sciences }\end{array}$ \\
\hline 17 & $\begin{array}{l}\text { Serapioni (2016). Conceitos e métodos para a avaliação de } \\
\text { programas sociais e políticas públicas. }\end{array}$ & $\begin{array}{c}\text { Revista da } \\
\text { Faculdade de } \\
\text { Letras da } \\
\text { Universidade do } \\
\text { Porto }\end{array}$ \\
\hline 13 & $\begin{array}{l}\text { Gasparini e Furtado (2014). Avaliação de Programas e } \\
\text { Serviços Sociais no Brasil: uma análise das práticas no } \\
\text { contexto atual. }\end{array}$ & $\begin{array}{l}\text { Serviço Social \& } \\
\text { Sociedade }\end{array}$ \\
\hline 11 & $\begin{array}{l}\text { Cortês-Neto et al., (2010). Elaboração de indicadores de } \\
\text { sucesso em programas de saúde pública com foco sócio- } \\
\text { esportivo. }\end{array}$ & $\begin{array}{l}\text { Revista Salud } \\
\text { Pública }\end{array}$ \\
\hline 7 & $\begin{array}{l}\text { Debray et al., (2014). Lessons from a Federal Grant for School } \\
\text { Diversity: Tracing a Theory of Change and Implementation of } \\
\text { Local Policies. }\end{array}$ & $\begin{array}{l}\text { Education Policy } \\
\text { Analysis Archives }\end{array}$ \\
\hline 6 & $\begin{array}{l}\text { Ribeiro e Ribeiro (2015). Saúde e Prevenção nas Escolas } \\
\text { (SPE): elementos para avaliação de projetos sociais em } \\
\text { Juazeiro, Bahia, Brasil. }\end{array}$ & $\begin{array}{c}\text { Interface - } \\
\text { Comunicação } \\
\text { Saúde Educação }\end{array}$ \\
\hline 5 & $\begin{array}{l}\text { Pospieszna (2014). Democracy assistance and women's } \\
\text { political empowerment in post-conflict countries. }\end{array}$ & Democratization \\
\hline 5 & $\begin{array}{l}\text { Ungar, Duque e Hernandez (2011). Can focus groups be used } \\
\text { for longitudinal evaluation? Findings from the Medellin early } \\
\text { prevention of aggression program. }\end{array}$ & $\begin{array}{l}\text { International } \\
\text { Journal of Multiple } \\
\text { Research } \\
\text { Approaches }\end{array}$ \\
\hline 4 & $\begin{array}{l}\text { Bahn e Giles (2012). Evaluation of the Neurodegenerative } \\
\text { Conditions Coordinated Care Program (NCCCP) in Western } \\
\text { Australia: Barriers to better service provision. }\end{array}$ & $\begin{array}{l}\text { Evaluation and } \\
\text { Program Planning }\end{array}$ \\
\hline 4 & $\begin{array}{l}\text { Gutiérrez et al., (2013). Effects of the Frontiers Prevention } \\
\text { Project in Ecuador on sexual behaviours and sexually } \\
\text { transmitted infections amongst men who have sex with men }\end{array}$ & $\begin{array}{l}\text { Journal of } \\
\text { Development } \\
\text { Effectiveness }\end{array}$ \\
\hline
\end{tabular}




\section{Quadro 2 - Quantidade de citações dos artigos encontrados na pesquisa.}

\begin{tabular}{|c|c|c|}
\hline & $\begin{array}{l}\text { and female sex workers: Challenges on evaluating complex } \\
\text { interventions. }\end{array}$ & \\
\hline 4 & $\begin{array}{l}\text { Cardoso (2014). O que a perspectiva antropológica tem a dizer } \\
\text { sobre a avaliação de projetos sociais apoiados pela cooperação } \\
\text { internacional? }\end{array}$ & $\begin{array}{l}\text { Horizontes } \\
\text { Antropológicos }\end{array}$ \\
\hline 2 & $\begin{array}{l}\text { McInnes et al., (2016). Improved Targeting of Social } \\
\text { Programs: An Application to a State Job Coaching Program for } \\
\text { Adults with Intellectual Disabilities. }\end{array}$ & $\begin{array}{c}\text { Eastern Economic } \\
\text { Journal }\end{array}$ \\
\hline 2 & $\begin{array}{l}\text { Newell e Graham (2012). Using an Empowerment Evaluation } \\
\text { Approach with Community-based Programs: Reflections from } \\
\text { the Front Line. }\end{array}$ & $\begin{array}{l}\text { Evaluation Journal } \\
\text { of Australasia }\end{array}$ \\
\hline 1 & $\begin{array}{l}\text { Zarychta et al., (2019). Assessing the impacts of governance } \\
\text { reforms on health services delivery: a quasi-experimental, } \\
\text { multi-method, and participatory approach. }\end{array}$ & $\begin{array}{l}\text { Health Services } \\
\text { and Outcomes } \\
\text { Research } \\
\text { Methodology }\end{array}$ \\
\hline 1 & $\begin{array}{l}\text { Menezes e Mourão (2017). Programa Minha Casa Minha Vida: } \\
\text { Sob a perspectiva da qualidade de vida. }\end{array}$ & Psicoperspectivas \\
\hline 1 & $\begin{array}{l}\text { Diniz e Voese (2016). Avaliação de programas sociais: Uma } \\
\text { análise da relação do programa bolsa família e o } \\
\text { desenvolvimento municipal. }\end{array}$ & Revista Espacios \\
\hline 1 & $\begin{array}{l}\text { Sauma et al., (2016). Design of a methodology for impact } \\
\text { assessment of energy efficiency programs: measuring indirect } \\
\text { effects in the Chilean case. }\end{array}$ & Energy Efficiency \\
\hline 0 & $\begin{array}{l}\text { Rêgo, Silva e Elliot (2017). Avaliação de Impacto Social da } \\
\text { Comunidade Vida e Paz: Um exercício de meta-avaliação. }\end{array}$ & Meta: Avaliação \\
\hline 0 & $\begin{array}{l}\text { Bennie et al., (2019). We were made to feel comfortable and } \\
\ldots \text { safe': co-creating, delivering, and evaluating coach } \\
\text { education and health promotion workshops with Aboriginal } \\
\text { Australian peoples }\end{array}$ & $\begin{array}{l}\text { Annals of Leisure } \\
\quad \text { Research }\end{array}$ \\
\hline 0 & $\begin{array}{l}\text { Gonzáles, Gómes e Prado (2019). Metodología de evaluación } \\
\text { de impacto aplicada al programa regional de apoyo al } \\
\text { emprendimiento de Corfo, Chile. }\end{array}$ & Interciencia \\
\hline 0 & $\begin{array}{l}\text { Andriola e Gomes (2017). Programa um computador por } \\
\text { aluno: Uma análise bilbiométrica. }\end{array}$ & Educar em Revista \\
\hline 0 & $\begin{array}{l}\text { Dugand e Brandão (2017). A Teoria da Mudança como } \\
\text { Ferramenta Avaliativa do Desenho dos Programas Sociais: o } \\
\text { caso das ações estruturantes para Comunidades Quilombolas. }\end{array}$ & Meta: Avaliação \\
\hline
\end{tabular}

Fonte: Elaborado pelas autoras.

No Quadro 3 são apresentadas as oito teses e oito dissertações resultantes da pesquisa. Nota-se que houve maior número de trabalhos publicados a respeito da avaliação de programas sociais nos últimos três anos pesquisados 2019, 2018 e 2017. Sob o ponto de vista dos programas de Pós-Graduação que 
mais publicaram sobre a avaliação de programas sociais, a Universidade de Viçosa e a Universidade de Brasília se destacaram, com três trabalhos cada uma.

\section{Quadro 3 - Teses e dissertações encontradas na base de dados Biblioteca Digital de Teses e Dissertações (BDTD) do MCTI que tratam da avaliação de programas sociais.}

\begin{tabular}{|c|c|c|c|}
\hline $\begin{array}{c}\text { Tipo de } \\
\text { documento }\end{array}$ & $\begin{array}{l}\text { Ano da } \\
\text { defesa }\end{array}$ & Título & Universidade \\
\hline Tese & $\begin{array}{l}\text { Rocha } \\
(2015)\end{array}$ & $\begin{array}{l}\text { Impacto do Programa Bolsa Família sobre } \\
\text { os padrões de consumo das famílias } \\
\text { Brasileiras }\end{array}$ & $\begin{array}{l}\text { Universidade } \\
\text { Federal de Viçosa }\end{array}$ \\
\hline Tese & $\begin{array}{l}\text { Barreto } \\
(2019)\end{array}$ & $\begin{array}{l}\text { Projeto QualiSus-rede: da análise } \\
\text { estratégica à percepção dos atores sobre os } \\
\text { desafios para a implementação das redes } \\
\text { de atenção à saúde no Brasil }\end{array}$ & $\begin{array}{l}\text { Universidade de } \\
\text { Brasília }\end{array}$ \\
\hline Tese & $\begin{array}{l}\text { Menezes } \\
(2017)\end{array}$ & $\begin{array}{l}\text { O impacto dos programas sociais na } \\
\text { qualidade de vida de seus participantes }\end{array}$ & $\begin{array}{l}\text { Universidade } \\
\text { Salgado de Oliveira }\end{array}$ \\
\hline Tese & $\begin{array}{l}\text { Taño } \\
(2017)\end{array}$ & $\begin{array}{l}\text { A constituição de ações intersetoriais de } \\
\text { atenção às crianças e adolescentes em } \\
\text { sofrimento psíquico }\end{array}$ & $\begin{array}{l}\text { Universidade } \\
\text { Federal de São } \\
\text { Carlos }\end{array}$ \\
\hline Tese & $\begin{array}{l}\text { Lelis } \\
(2016)\end{array}$ & $\begin{array}{l}\text { Transferência Condicional de Renda e } \\
\text { Políticas de Desenvolvimento Rural no } \\
\text { Brasil: Explorando Potenciais Sinergias } \\
\text { entre Bolsa Família e o Projeto Pró-Gavião }\end{array}$ & $\begin{array}{l}\text { Universidade } \\
\text { Federal de Viçosa }\end{array}$ \\
\hline Tese & $\begin{array}{l}\text { Gouveia } \\
(2013)\end{array}$ & $\begin{array}{l}\text { Avaliação da eficácia e efetividade do } \\
\text { programa de erradicação do trabalho } \\
\text { infantil a partir da perspectiva dos usuários } \\
\text { e agentes }\end{array}$ & $\begin{array}{l}\text { Universidade } \\
\text { Federal da Paraíba }\end{array}$ \\
\hline Tese & $\begin{array}{l}\text { Silva } \\
(2018)\end{array}$ & $\begin{array}{l}\text { Avaliação da implementação do plano de } \\
\text { mobilidade urbana em Natal - RN }\end{array}$ & $\begin{array}{l}\text { Universidade } \\
\text { Federal do Rio } \\
\text { Grande do Norte }\end{array}$ \\
\hline Tese & $\begin{array}{l}\text { Kinpara } \\
(2013)\end{array}$ & $\begin{array}{l}\text { Abordagem Multinível na Avaliação do } \\
\text { Programa Bolsa Família }\end{array}$ & $\begin{array}{l}\text { Universidade de } \\
\text { Brasília }\end{array}$ \\
\hline Dissertação & $\begin{array}{l}\text { Cilento } \\
(2019)\end{array}$ & $\begin{array}{l}\text { Gestão de projetos no terceiro setor: } \\
\text { avaliação de projetos sociais na economia } \\
\text { solidária }\end{array}$ & $\begin{array}{l}\text { Universidade } \\
\text { Federal da Paraíba }\end{array}$ \\
\hline Dissertação & $\begin{array}{l}\text { Breyer } \\
(2017)\end{array}$ & $\begin{array}{l}\text { Programa Segundo Tempo: um estudo } \\
\text { sobre os objetivos e os processos de } \\
\text { avaliação e de construção de indicadores de } \\
\text { impacto }\end{array}$ & $\begin{array}{l}\text { Universidade } \\
\text { Federal do Rio } \\
\text { Grande do Sul }\end{array}$ \\
\hline Dissertação & $\begin{array}{l}\text { Birman } \\
(2016)\end{array}$ & $\begin{array}{l}\text { Avaliação de egressos de projetos sociais: o } \\
\text { que sabemos sobre o pós-projeto? }\end{array}$ & $\begin{array}{l}\text { Universidade } \\
\text { Federal do Rio de } \\
\text { Janeiro }\end{array}$ \\
\hline Dissertação & $\begin{array}{l}\text { Silva } \\
(2015)\end{array}$ & $\begin{array}{l}\text { Construção e validação de instrumentos de } \\
\text { avaliação dos projetos sociais do Programa } \\
\text { Petrobrás Socioambiental }\end{array}$ & $\begin{array}{l}\text { Fundação } \\
\text { Cesgranrio }\end{array}$ \\
\hline
\end{tabular}




\section{Quadro 3 - Teses e dissertações encontradas na base de dados Biblioteca Digital de Teses e Dissertações (BDTD) do MCTI que tratam da avaliação de programas sociais.}

\begin{tabular}{|c|c|l|l|}
\hline Dissertação & $\begin{array}{c}\text { Neves } \\
(2015)\end{array}$ & Fatores para avaliação de projetos sociais & $\begin{array}{l}\text { Universidade } \\
\text { Federal de } \\
\text { Pernambuco }\end{array}$ \\
\hline Dissertação & $\begin{array}{c}\text { Vasconcelo } \\
\text { s (2014) }\end{array}$ & $\begin{array}{l}\text { Projeto Criança Feliz: um estudo de caso da } \\
\text { aplicação da cosmovisão calvinista de ação } \\
\text { social }\end{array}$ & $\begin{array}{l}\text { Universidade } \\
\text { Presbiteriana } \\
\text { Mackenzie }\end{array}$ \\
\hline Dissertação & $\begin{array}{c}\text { Moreira } \\
(2014)\end{array}$ & $\begin{array}{l}\text { Externalidades do Programa Bolsa Família } \\
\text { sobre a violência doméstica contra a mulher } \\
\text { no Brasil }\end{array}$ & $\begin{array}{l}\text { Universidade } \\
\text { Federal de Viçosa }\end{array}$ \\
\hline Dissertação & $\begin{array}{c}\text { Jreige } \\
(2013)\end{array}$ & $\begin{array}{l}\text { Programa de Residência Multiprofissional } \\
\text { em Saúde: Um estudo sobre os efeitos na } \\
\text { crença, satisfação e comprometimento }\end{array}$ & $\begin{array}{l}\text { Universidade de } \\
\text { Brasília }\end{array}$ \\
\hline
\end{tabular}

Fonte: Elaborado pelas autoras.

\section{Resultados e discussões}

Nesta seção são apresentados os resultados da pesquisa. Primeiramente tem-se dados descritivos dos trabalhos publicados nas bases de dados selecionadas que tratam da avaliação de impacto e, posteriormente, as metodologias de avaliação de programas sociais utilizadas nos trabalhos.

\subsection{Metodologias de avaliação de projetos sociais}

As metodologias de avaliação de impacto de programas sociais podem contribuir para o entendimento das mudanças das condições de vida dos beneficiários, auxiliando a compreender quais os resultados estão sendo alcançados.

A combinação de métodos de avaliação é uma estratégia útil para ajudar a compensar as deficiências que podem ser encontradas ao se usar um único método de avaliação. Os métodos de avaliação mistos são agora comuns. No passado, utilizavam-se métodos de avaliação qualitativos ou quantitativos, mas hoje em dia também se incluem combinações dentro de categorias quantitativas e qualitativas, fazendo com que a avaliação se torne mais completa (ASSUMPÇÃO; CAMPOS, 2011; SERAPIONI, 2016; STERN et al., 2012).

De um ponto de vista técnico, quando se quer avaliar o impacto de um programa, é necessário comparar a experiência de dois grupos: o grupo de tratamento, que engloba os participantes/usuários do programa, e o grupo de controle, que inclui pessoas não participantes do projeto. "O grupo de controle é considerado um elemento chave para gerar argumentos em favor da relação de causa e efeito entre o projeto (ou negócio) e as mudanças na realidade de seus beneficiários, ou usuários, provocadas por causas diversas." (FABIANI et al., 2018, p. 9). Sendo assim, os métodos de avaliação de impacto são classificados pela forma como se criam os grupos de controle (Quadro 4). 


\section{Quadro 4 - Classes de métodos de avaliação de impacto.}

\begin{tabular}{|c|c|c|}
\hline $\begin{array}{l}\text { Classes de } \\
\text { métodos }\end{array}$ & Composição & Aplicação \\
\hline $\begin{array}{l}\text { Método } \\
\text { experimental }\end{array}$ & $\begin{array}{l}\text { Os sujeitos são escolhidos para } \\
\text { ser parte do grupo de controle } \\
\text { ou do grupo de tratamento de } \\
\text { modo aleatório. }\end{array}$ & $\begin{array}{l}\text { Utilizado para avaliar intervenções de } \\
\text { elevado porte, como políticas ou } \\
\text { programas públicos estaduais, que } \\
\text { requerem coleta de dados primários em } \\
\text { larga escala. }\end{array}$ \\
\hline $\begin{array}{l}\text { Métodos quase } \\
\text { experimental }\end{array}$ & $\begin{array}{l}\text { São estabelecidos grupos de } \\
\text { comparação por meio de } \\
\text { técnicas de pareamento, } \\
\text { baseados em particularidades } \\
\text { verificadas nos grupos de } \\
\text { controle e tratamento. }\end{array}$ & $\begin{array}{l}\text { Dependem de demais métodos para } \\
\text { ciência do que teria acontecido se a } \\
\text { intervenção fosse aplicada, considerando } \\
\text { que é impossível a alocação aleatória para } \\
\text { grupos de tratamento. }\end{array}$ \\
\hline $\begin{array}{l}\text { Método não } \\
\text { experimental. }\end{array}$ & $\begin{array}{l}\text { O grupo de controle é hipotético } \\
\text { ou não é usado um grupo de } \\
\text { comparação. }\end{array}$ & $\begin{array}{l}\text { Os métodos não experimentais são } \\
\text { aplicados quando não é possível ou } \\
\text { desejável aplicar as abordagens } \\
\text { experimentais e quase experimentais } \\
\text { devido à limitação de recursos ou falta de } \\
\text { desejo do investidor social para se } \\
\text { delinear tais grupos. Nesses casos, a } \\
\text { avaliação de impacto utiliza dados } \\
\text { hipotéticos ou lógicos, estimando o que } \\
\text { aconteceria se não existisse a intervenção, } \\
\text { não havendo necessidade de um grupo de } \\
\text { comparação. }\end{array}$ \\
\hline
\end{tabular}

Fonte: Elaborado a partir de Fabiani et al. (2018).

Fabiani et al. (2018) explicam ainda que a escolha da metodologia de avaliação deve considerar fatores como os recursos disponíveis e as restrições, o período da intervenção, o prazo para a finalização da avaliação de impacto, vivências, informações adquiridas e as normas da organização responsável pelo projeto. As características do projeto e da instituição que está realizando a intervenção e o estágio do projeto precisam ser compreendidos para que a escolha da metodologia seja acertada. E por fim, é preciso levar em conta também a finalidade da avaliação, o que será respondido e quais serão os stakeholders envolvidos na avaliação.

Bengo et al. (2016) reiteram este pensamento ao afirmar que é preciso considerar que diferentes categorias de stakeholders envolvidos na avaliação de programas sociais têm objetivos distintos e às vezes contrastantes. Com base nesses objetivos, também têm necessidades de informações diferentes. Portanto, a organização deve mapear as expectativas, objetivos e interesses de diferentes stakeholders a fim de desenvolver um conjunto de indicadores e métricas.

Entre os stakeholders citados por Bengo et al. (2016), existem os financiadores e doadores, os formuladores de políticas públicas e os empreendedores sociais. Jannuzzi $(2011,2014)$ reforça que os responsáveis por 
elaborar políticas públicas podem fazer uso de indicadores e métricas na avaliação das intervenções, no âmbito das comunidades e do governo.

Complementando, Bengo et al. (2016) destacam três grupos de metodologias de avaliação que podem atender aos anseios dos financiadores de programas sociais:

1) Metodologias que levam à construção de um indicador sintético destinado a medir a criação de valor social. Estas metodologias medem o valor dos benefícios sociais criados por uma organização em relação ao custo do atingimento desses benefícios;

2) Abordagens baseadas em metodologias que se concentram no processo de produção de um serviço ou produto social, articulando a análise do desempenho social dos negócios em entradas, saídas, resultados e impactos;

3) Metodologias baseadas em dashboards e scorecards, que incluem métodos destinados a fornecer uma imagem dos resultados de acordo com diferentes dimensões de desempenho.

A partir disso, comprova-se que as metodologias de avaliação de impactos apontadas na literatura são importantes meios de auxiliar os gestores e os investidores nas tomadas de decisões, já que oferecem informações necessárias para aprimorar o processo de planejamento e de gestão dos seus programas. Portanto, estes stakeholders necessitam de conhecimento sobre as metodologias de avaliação de impacto de programas sociais existentes, para que possam utilizá-las para medir os impactos dos programas sociais que desenvolvem.

Algumas instituições desempenham um papel fundamental na disseminação do conhecimento sobre as metodologias de avaliação de impacto. Em outubro de 2007, a Fundação Rockefeller juntou um grupo diversificado de investidores para discutir formas de apoiar projetos visando retornos financeiros, sociais e ambientais. O resultado desse encontro culminou na elaboração de um catálogo de abordagens de avaliação de impacto chamado "Catalog of approaches to impact measurement: Assessing social impact in private ventures" (OLSEN e GALIMIDI, 2008).

Neste documento, as metodologias de avaliação de impacto são classificadas em três grupos, sendo:

1. Sistemas de classificação: a partir de um conjunto fixo de indicadores, a qualidade do investimento de impacto é resumida por uma pontuação ou símbolo;

2. Sistema de avaliação: a partir de um conjunto fixo ou personalizado de indicadores, são avaliadas as características, práticas e resultados dos investimentos, mas não são fornecidas as ferramentas explícitas para gerenciar o rastreamento de dados operacionais pela organização ao longo do tempo;

3. Sistema de gerenciamento: fornece ferramentas para as organizações gerenciarem informações operacionais detalhadas sobre os fatores de impacto.

No Quadro 5 estão apontadas as metodologias de avaliação de impacto descritas por Olsen e Galimidi (2008) de acordo com cada grupo. 


\section{Quadro 5 - Metodologias de avaliação de impacto classificadas por grupo.}

\begin{tabular}{|c|c|c|}
\hline Sistemas de classificação & Sistema de Avaliação & Sistema de gerenciamento \\
\hline Fair Trade Certification & Ecological Footprint & $\begin{array}{l}\text { Balanced Scorecard modified } \\
\text { to include impact }\end{array}$ \\
\hline Ecological Footprint & $\begin{array}{l}\text { Research Indicators for } \\
\text { Sustainable Employment }\end{array}$ & Trucost \\
\hline $\begin{array}{l}\text { Leadership in Energy \& } \\
\text { Environmental Design }\end{array}$ & Social Impact Assessment & SROI Toolkit \\
\hline $\begin{array}{l}\text { Compass Investment } \\
\text { Sustainability Assessment }\end{array}$ & $\begin{array}{l}\text { Compass Investment } \\
\text { Sustainability Assessment }\end{array}$ & SROI Framework \\
\hline Dalberg Approach & SROI Analysis & SROI Lite \\
\hline Charity Analysis Tool & Dalberg Approach & $\begin{array}{l}\text { Portfolio Data Management } \\
\text { System }\end{array}$ \\
\hline Social Rating & SROI Toolkit & \\
\hline B Rating System & Charity Analysis Tool & \\
\hline $\begin{array}{l}\text { Human Impact }+ \\
\text { ProfitFramework }\end{array}$ & SROI Calculator & \\
\hline \multirow[t]{10}{*}{ Political Return of Investment } & SROI Framework & \\
\hline & $\begin{array}{l}\text { Development Outcome } \\
\text { Tracking System }\end{array}$ & \\
\hline & Progress out of Poverty Index & \\
\hline & Social Rating & \\
\hline & $\begin{array}{l}\text { Environmental Performance } \\
\text { Reporting System }\end{array}$ & \\
\hline & $\begin{array}{l}\text { Human Impact + Profit } \\
\text { Framework }\end{array}$ & \\
\hline & $\begin{array}{l}\text { Movement Above the US } \\
\$ 1 / \text { Day Threshold }\end{array}$ & \\
\hline & $\begin{array}{l}\text { Portfolio Data Management } \\
\text { System }\end{array}$ & \\
\hline & $\begin{array}{l}\text { Political Return on } \\
\text { Investment }\end{array}$ & \\
\hline & Social Value Metrics & \\
\hline
\end{tabular}

Fonte: Olsen e Galimidi (2008).

Neste estudo, a classe de metodologias estudada é a segunda, "sistemas de avaliação", pois por meio destas metodologias são avaliadas as características, práticas e resultados dos investimentos em programas sociais (OLSEN e GALIMIDI, 2008). As metodologias relacionadas aos programas 
sociais apontadas no Catálogo por Olsen e Galimidi como aplicáveis ao Brasil são: a Development Tracking System (DOTS) e a Social Return on Investment (SROI).

Por sua vez, Fabiani et al. (2018) identificam as seguintes metodologias: Social Return on Investment (SROI), Difference in differences (DID), Matching (Propensity Score Matching), Regression Discontinuity Design (RDD), Instrumental Variables (IV) e Randomized Control Trials (RCT).

O Quadro 6 resume as metodologias citadas, identificando a classe do método e o instrumento de medição.

\section{Quadro 6 - Metodologias de avaliação de programa sociais segundo vários autores.}

\begin{tabular}{|c|c|c|c|}
\hline Metodologia & Objetivo & $\begin{array}{l}\text { Classe de } \\
\text { método }\end{array}$ & $\begin{array}{l}\text { Instru- } \\
\text { mento de } \\
\text { medição }\end{array}$ \\
\hline $\begin{array}{l}\text { Development } \\
\text { Tracking System } \\
\text { (DOTS) }\end{array}$ & $\begin{array}{l}\text { Objetiva comparar o desempenho de } \\
\text { programas sociais e empresas que usam o } \\
\text { trabalho de consultoria da International } \\
\text { Finance Corporation (IFC) em todos os } \\
\text { continentes. } \\
\text { A metodologia pode ser usada para que a } \\
\text { IFC monitore cada projeto de perto e } \\
\text { resolva imediatamente quaisquer } \\
\text { problemas que possam surgir (OLSEN e } \\
\text { GALIMIDI, 2008; VELDMAN, 2009; } \\
\text { INTERNATIONAL FINANCE CORPORATION, } \\
\text { 2016). }\end{array}$ & $\begin{array}{l}\text { Método não } \\
\text { experimen- } \\
\text { tal }\end{array}$ & $\begin{array}{l}\text { Indicador } \\
\text { sintético }\end{array}$ \\
\hline $\begin{array}{l}\text { Social Return of } \\
\text { Investment (SROI) }\end{array}$ & $\begin{array}{l}\text { Objetiva medir o valor dos benefícios sociais } \\
\text { criados por uma organização em relação ao } \\
\text { custo da intervenção (BENGO et al., 2016; } \\
\text { BRANDÃO et al., 2014). A metodologia } \\
\text { pode ser usada como uma ferramenta para } \\
\text { planejamento e aprimoramento } \\
\text { estratégico, para comunicar impacto e } \\
\text { atrair investimento ou para tomar decisões } \\
\text { de investimento. }\end{array}$ & $\begin{array}{l}\text { Método não } \\
\text { experimen- } \\
\text { tal }\end{array}$ & $\begin{array}{l}\text { Indicador } \\
\text { sintético }\end{array}$ \\
\hline $\begin{array}{l}\text { Difference in } \\
\text { Differences (DID) }\end{array}$ & $\begin{array}{l}\text { Objetiva comparar o grupo de tratamento } \\
\text { com um grupo semelhante que existe em } \\
\text { outro lugar e que não é criado por meio de } \\
\text { randomização. É uma técnica que requer } \\
\text { que as características dos dois grupos } \\
\text { sejam homogêneas (VELDMAN, 2009). } \\
\text { Athey e Imbens (2006) explicam que esse } \\
\text { método é usado em problemas com várias } \\
\text { subpopulações - algumas sujeitas a } \\
\text { intervenção, ou grupo de tratamento, e } \\
\text { outras não, grupo de controle - e os } \\
\text { resultados são medidos em cada grupo } \\
\text { antes e depois da intervenção. Para explicar } \\
\text { as mudanças ao longo do tempo, a } \\
\text { mudança experimentada pelo grupo de }\end{array}$ & $\begin{array}{l}\text { Método } \\
\text { quase- } \\
\text { experimen- } \\
\text { tal }\end{array}$ & $\begin{array}{l}\text { Dashboards } \\
\text { e scorecards }\end{array}$ \\
\hline
\end{tabular}




\section{Quadro 6 - Metodologias de avaliação de programa sociais segundo vários autores.}

\begin{tabular}{|c|c|c|c|}
\hline & $\begin{array}{l}\text { tratamento é relacionada à mudança } \\
\text { experimentada pelo grupo de controle. }\end{array}$ & & \\
\hline $\begin{array}{l}\text { Propensity Score } \\
\text { Matching (PSM) }\end{array}$ & $\begin{array}{l}\text { Objetiva designar um grupo para um } \\
\text { tratamento específico, dado um vetor de } \\
\text { covariáveis observadas. A randomização } \\
\text { elimina possíveis vieses. Desta forma, no } \\
\text { desfecho do projeto ou intervenção, a } \\
\text { diferença entre os grupos não será atribuída } \\
\text { a fatores externos ou a outras variáveis } \\
\text { observadas (ROSENBAUM e RUBIN, 1983). }\end{array}$ & $\begin{array}{l}\text { Método } \\
\text { experimen- } \\
\text { tal }\end{array}$ & $\begin{array}{l}\text { Dashboards } \\
\text { e scorecards }\end{array}$ \\
\hline $\begin{array}{l}\text { Regression } \\
\text { Discontinuity } \\
\text { Design (RDD) }\end{array}$ & $\begin{array}{l}\text { Objetiva criar um critério de corte que } \\
\text { garante o tratamento a grupos pré- } \\
\text { determinados com base em uma pontuação } \\
\text { bem definida e conhecida dos participantes. } \\
\text { O RDD classifica os indivíduos com base em } \\
\text { critérios mensuráveis específicos. O grupo } \\
\text { de controle é composto por indivíduos que } \\
\text { estão próximos do ponto de corte mas que } \\
\text { caem no lado "errado" desse ponto de corte } \\
\text { e, portanto, não recebem o programa. O } \\
\text { grupo do tratamento é composto por } \\
\text { pessoas que estavam próximas ao ponto de } \\
\text { corte, mas que caem do lado "correto". Isso } \\
\text { permite que as variáveis de critério sejam } \\
\text { muito semelhantes entre os participantes e } \\
\text { os não participantes e, portanto, essas } \\
\text { variáveis são controladas (VELMAN, 2009; } \\
\text { IMBENS e LEMIEUX, } 2008 \text {; PINTO, 2012). } \\
\text { Este critério de corte implica a grande } \\
\text { vantagem dos projetos de RDD, já que este } \\
\text { método pode ser apropriado quando se } \\
\text { quer direcionar um programa ou } \\
\text { tratamento àqueles que mais precisam ou } \\
\text { merecem, como por exemplo, o } \\
\text { oferecimento de reforço escolar a alunos } \\
\text { que tiveram notas abaixo da média. }\end{array}$ & $\begin{array}{l}\text { Método } \\
\text { experimen- } \\
\text { tal }\end{array}$ & $\begin{array}{l}\text { Dashboards } \\
\text { e scorecards }\end{array}$ \\
\hline $\begin{array}{l}\text { Instrumental } \\
\text { Variable (IV) }\end{array}$ & $\begin{array}{l}\text { Objetiva estimar relações causais quando } \\
\text { não é possível realizar experimentos } \\
\text { controlados ou quando um tratamento não } \\
\text { é entregue com sucesso em um } \\
\text { experimento randomizado. O método é } \\
\text { baseado em variáveis não observáveis pelo } \\
\text { pesquisador que influenciam a decisão de } \\
\text { fazer parte ou não de um programa, o que } \\
\text { poderia prejudicar o resultado (PINTO, } \\
2012 \text { ). Esta metodologia é utilizada para } \\
\text { selecionar os participantes de projetos } \\
\text { sociais quando métodos de pareamento ou } \\
\text { outros métodos de escolha não são } \\
\text { possíveis, então, é contado com o fator } \\
\text { "sorte", como por exemplo um sorteio. }\end{array}$ & $\begin{array}{l}\text { Método } \\
\text { quase- } \\
\text { experimen- } \\
\text { tal }\end{array}$ & $\begin{array}{l}\text { Dashboards } \\
\text { e scorecards }\end{array}$ \\
\hline
\end{tabular}




\section{Quadro 6 - Metodologias de avaliação de programa sociais segundo vários autores.}

\begin{tabular}{|l|l|l|l|}
\hline Randomized & $\begin{array}{l}\text { Objetiva medir e comparar resultados } \\
\text { Control Trials (RCT) } \\
\text { aplicados aos participantes de um programa } \\
\text { ou projeto com um grupo de controle que } \\
\text { não participou da intervenção. Na seleção } \\
\text { de participantes do programa é considerada } \\
\text { alguma característica em comum. (WHITE, } \\
\text { 2013). }\end{array}$ & $\begin{array}{l}\text { Dashboards } \\
\text { tal }\end{array}$ \\
Em vez disso, os participantes são \\
selecionados por meio de escolha e auto \\
seleção ao programa. A escolha se refere ao \\
fato de que a agência ou empresa \\
implementa a intervenção em \\
subpopulações específicas, como famílias \\
chefiadas por mulheres, pequenas \\
empresas, crianças em risco, escolas em \\
distritos pobres assim por diante. A auto \\
seleção ocorre porque as pessoas \\
raramente são coagidas a participar de \\
programas de desenvolvimento. Eles o \\
fazem voluntariamente, aqueles que \\
decidem participar podem ter \\
características diferentes daqueles que não \\
ofazem.
\end{tabular} \mid

Fonte: Elaborado pelas autoras.

Apesar da avaliação de impacto de programas sociais ser um tema recorrente na literatura, este tipo de avaliação ainda não foi incorporado ao cotidiano de um grande número de OSCs no Brasil. Em parte, isto se dá devido à falta de conhecimento dos gestores destas organizações sobre as metodologias existentes, em parte pela complexidade inerente às metodologias de avaliação (JANNUZZI, 2011). Entretanto, Fabiani et al. (2018) alertam que, apesar dos problemas e custos envolvidos, a mensuração do impacto de programas sociais é o que garante a efetividade das intervenções e sua contribuição para uma sociedade mais sustentável.

3.2 Metodologias de avaliação de programas sociais apresentadas na produção científica

Esta seção apresenta os resultados da seleção dos trabalhos que aplicam metodologias de avaliação em programas sociais. Dos 41 trabalhos encontrados na bibliométrica, 22 mencionam algum tipo de metodologia de avaliação de programas sociais, como mostrado no Quadro 7.

Neste quadro pode ser observado que o ano de 2017 registrou o maior número de publicações: três artigos, uma tese e uma dissertação. Por outro lado, não houve publicações, nas bases selecionadas, sobre o tema nos anos 2010 e 2018. 


\section{Quadro 7 - Metodologias e ferramentas de pesquisa adotados nos trabalhos.}

\begin{tabular}{|c|c|c|}
\hline Tipo & Autor e ano & Metodologia ou técnica de avaliação \\
\hline Artigo & $\begin{array}{l}\text { Zarychta et al. } \\
(2019)\end{array}$ & $\begin{array}{l}\text { Abordagem quase experimental combinada com uma análise } \\
\text { de diferença nas diferenças (DID), abordagem multi métodos } \\
\text { que engloba a compilação de dados, pesquisa domiciliar e } \\
\text { entrevistas semiestruturadas e processo participativo de } \\
\text { avaliação de políticas. }\end{array}$ \\
\hline Artigo & $\begin{array}{l}\text { Gonzáles, } \\
\text { Gómes e Prado } \\
(2019)\end{array}$ & $\begin{array}{l}\text { Análise estatística. } \\
\text { Esta não é uma metodologia caracterizada com uma } \\
\text { metodologia de avaliação de impacto, mas permite avaliar o } \\
\text { programa social. }\end{array}$ \\
\hline Artigo & $\begin{array}{l}\text { Rêgo, Silva e } \\
\text { Elliot (2017) }\end{array}$ & Social Return on Investment (SROI). \\
\hline Artigo & $\begin{array}{l}\text { Menezes e } \\
\text { Mourão (2017) }\end{array}$ & $\begin{array}{l}\text { Escala Brasileira de Qualidade de Vida. } \\
\text { Esta não é uma metodologia caracterizada com uma } \\
\text { metodologia de avaliação de impacto, mas permite avaliar o } \\
\text { programa social. }\end{array}$ \\
\hline Artigo & $\begin{array}{l}\text { Dugand e } \\
\text { Brandão (2017) }\end{array}$ & Teoria da Mudança. \\
\hline Tese & Menezes (2017) & $\begin{array}{l}\text { Escala brasileira de qualidade de vida e comparação de } \\
\text { grupos. } \\
\text { Esta não é uma metodologia caracterizada com uma } \\
\text { metodologia de avaliação de impacto, mas permite avaliar o } \\
\text { programa social. }\end{array}$ \\
\hline Dissertação & Breyer (2017) & $\begin{array}{l}\text { Qualitativa: entrevistas semiestruturadas e análise de } \\
\text { conteúdo. } \\
\text { Esta não é uma metodologia caracterizada com uma } \\
\text { metodologia de avaliação de impacto, mas permite avaliar o } \\
\text { programa social. }\end{array}$ \\
\hline Artigo & $\begin{array}{l}\text { Sauma et al. } \\
(2016)\end{array}$ & $\begin{array}{l}\text { Uma metodologia com foco especial nos efeitos indiretos. Para } \\
\text { medir esses efeitos indiretos usam-se três eixos: presença, } \\
\text { avaliação e capacidade de mobilização. } \\
\text { Esta não é uma metodologia caracterizada com uma } \\
\text { metodologia de avaliação de impacto, mas permite avaliar o } \\
\text { programa social. }\end{array}$ \\
\hline Artigo & $\begin{array}{l}\text { Serapioni } \\
(2016)\end{array}$ & $\begin{array}{l}\text { Positivista experimental, pragmatista da qualidade, } \\
\text { construtivista, Teoria da Mudança e avaliação realista. } \\
\text { Esta não é uma metodologia caracterizada com uma } \\
\text { metodologia de avaliação de impacto, mas permite avaliar o } \\
\text { programa social. }\end{array}$ \\
\hline Tese & Lelis (2016) & $\begin{array}{l}\text { Os métodos utilizados foram de natureza quase-experimentais } \\
\text { e foram empregados por meio de duas etapas: o Propensity } \\
\text { Score Matching e o método da Dupla Diferença e Efeitos Fixos. }\end{array}$ \\
\hline
\end{tabular}




\section{Quadro 7 - Metodologias e ferramentas de pesquisa adotados nos trabalhos.}

\begin{tabular}{|c|c|c|}
\hline Dissertação & Silva (2015) & $\begin{array}{l}\text { Lista de Verificação que contém } 94 \text { itens inspirados nas } \\
\text { obrigações contratuais, nas diretrizes do Programa Petrobras } \\
\text { Socioambiental e em padrões de qualidade de projetos já } \\
\text { reconhecidos na literatura. } \\
\text { Esta não é uma metodologia caracterizada com uma } \\
\text { metodologia de avaliação de impacto, mas permite avaliar o } \\
\text { programa social. }\end{array}$ \\
\hline Artigo & $\begin{array}{l}\text { Ahmadia et al. } \\
\text { (2015) }\end{array}$ & Comparação de grupos com abordagem quase experimental. \\
\hline Tese & Rocha (2015) & Propensity Score Matching e Diferenças em Diferenças. \\
\hline Dissertação & $\begin{array}{l}\text { Vasconcelos } \\
(2014)\end{array}$ & $\begin{array}{l}\text { Manual de Avaliação de Projetos Sociais da autoria de Eduardo } \\
\text { Marino, do Instituto Ayrton Senna. } \\
\text { Esta não é uma metodologia caracterizada com uma } \\
\text { metodologia de avaliação de impacto, mas permite avaliar o } \\
\text { programa social. }\end{array}$ \\
\hline Dissertação & Moreira (2014) & $\begin{array}{l}\text { Método econométrico (técnica Propensity Score e método de } \\
\text { Kernel). } \\
\text { Esta não é uma metodologia caracterizada com uma } \\
\text { metodologia de avaliação de impacto, mas permite avaliar o } \\
\text { programa social. }\end{array}$ \\
\hline Artigo & $\begin{array}{l}\text { Gutiérrez et al. } \\
(2013)\end{array}$ & $\begin{array}{l}\text { Um estudo comunitário, com distribuição aleatória de grupos } \\
\text { de intervenção e comparação e pesquisas transversais } \\
\text { repetidas. } \\
\text { Esta não é uma metodologia caracterizada com uma } \\
\text { metodologia de avaliação de impacto, mas permite avaliar o } \\
\text { programa social. }\end{array}$ \\
\hline Tese & Kinpara (2013) & $\begin{array}{l}\text { Análise de regressão multinível com medidas repetidas. Esta } \\
\text { não é uma metodologia caracterizada com uma metodologia } \\
\text { de avaliação de impacto, mas permite avaliar o programa } \\
\text { social. }\end{array}$ \\
\hline Tese & $a(2013)$ & $\begin{array}{l}\text { Foram realizados três estudos compostos por entrevistas, } \\
\text { análise de Conteúdo de Bardin e Análise Fatorial Comum (AFC) } \\
\text { através da técnica Componentes Principais ( } P C) \text {, com rotação } \\
\text { Varimax. }\end{array}$ \\
\hline Dissertação & Jreige (2013) & $\begin{array}{l}\text { Estudo longitudinal - Verificou-se o efeito da intervenção, } \\
\text { comparando-se os profissionais da saúde que participavam do } \\
\text { programa em diferentes estágios. }\end{array}$ \\
\hline Artigo & Lillis (2012) & $\begin{array}{l}\text { Autoestudo com revisão por pares, que se concentra em: (a) } \\
\text { autoavaliação interna das atividades; (b) relatório de } \\
\text { autoestudo; (c) processo de revisão por pares e (d) } \\
\text { implementação de recomendações da revisão por pares e } \\
\text { outras melhorias identificadas. } \\
\text { Esta não é uma metodologia caracterizada com uma } \\
\text { metodologia de avaliação de impacto, mas permite avaliar o } \\
\text { programa social. }\end{array}$ \\
\hline
\end{tabular}




\section{Quadro 7 - Metodologias e ferramentas de pesquisa adotados nos trabalhos.}

\begin{tabular}{|c|l|l|}
\hline Artigo & Cabral (2011) & Mapa de bens públicos (MBP). \\
\hline Artigo & $\begin{array}{l}\text { Ungar, Duque e } \\
\text { Hernandez } \\
(2011)\end{array}$ & $\begin{array}{l}\text { Comparação de grupos - avaliação quase experimental } \\
\text { utilizando grupos focais. }\end{array}$ \\
\hline
\end{tabular}

Fonte: Elaborado pelas autoras.

Em síntese, as metodologias de avaliação de impacto de programas sociais utilizadas nos trabalhos selecionados são: comparação de grupos com abordagem quase experimental, Mapa de bens públicos (MBP), Social Return on Investment (SROI), Teoria da Mudança, Propensity Score Matching, Diferenças em diferenças (DID), Componentes Principais (PC) com rotação Varimax e estudo longitudinal.

No quadro 7 foram descritas também outras metodologias não caracterizadas com metodologias de avaliação de impacto, mas que foram utilizadas para acompanhar ou avaliar os programas sociais. Esta variedade de metodologias parece refletir o pensamento de Jannuzzi (2011) de que modelos e prescrições padronizadas de avaliação de programas sociais podem trazer dificuldades aos avaliadores, sendo, nestes casos, preferível realizar uma avaliação personalizada, levando em consideração a maturidade do programa social.

\section{Considerações finais}

A pesquisa revelou que as metodologias de avaliação são ferramentas que contribuem com o cumprimento dos objetivos e maturidade dos programas sociais das organizações da sociedade civil. A sociedade tem legitimado a importância das metodologias de avaliação. Pode-se deduzir que um melhor entendimento da aplicação das ferramentas na avaliação dos resultados dos programas sociais pode ser um caminho para dar respostas às necessidades sociais.

Existe, de fato, o potencial para que os gestores e financiadores das OSCs mensurem o impacto dos programas sociais perseguindo a maturidade do programa e credibilidade junto aos stakeholders.

Considera-se necessário o desenvolvimento de novos estudos acadêmicos a respeito da avaliação de programas sociais que funcionem como catalisadores dos esforços no que tange à aplicação das metodologias no processo das intervenções.

Também há indícios de que o avanço do conhecimento científico a respeito das metodologias de avaliação de programas sociais pode qualificar o que poderia ser um desejado porvir para os gestores e financiadores dessas iniciativas. Logo, parece necessário que as instituições encontrem formas de assumir de forma efetiva, consistente e sistemática o conhecimento disponível na literatura a respeito das metodologias de avaliação. 


\section{REFERÊNCIAS}

AHMADIA, Gabby N.; GLEW, Louise; PROVOST, Mikaela; GILL, David; HIDAYAT, Nur Ismu; MANGUBHAI, Sangeeta Mangubhai. Integrating impact evaluation in the design and implementation of monitoring marine protected areas. Philosophical Transactions of the Royal Society B. Biological Sciences, v. 370, n. 1681, 2015.

ANDRIOLA, Wagner Bandeira; GOMES, Carlos Adriano Santos. Programa um computador por aluno (PROUCA): uma análise bibliométrica. Educar em Revista, Curitiba, n. 63, p. 267-288, 2017.

ASSUMPÇÃO, Jairo José; CAMPOS, Lucila Maria de Souza. Avaliação de projetos sociais em ONGs da Grande Florianópolis: um estudo sobre modelos relacionados ao foco de atuação. Revista de Administração Pública. v. 45, n. 1 , p. 209-242, 2011.

ATHEY, Susan.; IMBENS, Guido Wilhelmus. Identification and inference in nonlinear difference-in-differences models. Econométrica. v. 74, n. 2, p. 431497, 2006.

BAHN, Susanne; GILES, Margaret. Evaluation of the Neurodegenerative Conditions Coordinated Care Program (NCCCP) in Western Australia: Barriers to better service provision. Evaluation and Program Planning. v. 35, n. 1 , p. $40-46,2012$.

BARRETO, Jorge Otávio Maia. Projeto QualisuS-Rede: da análise estratégica à percepção dos atores sobre os desafios para a implementação das redes de atenção à saúde no Brasil. 2019. Tese (Doutorado em Saúde Coletiva) Universidade de Brasília, Brasília, 2019.

BENGO, Irene; ARENA, Marika; AZZONE, Giovanni; CALDERINI, Mario. Indicators and metrics for social business: a review of current approaches. Journal of Social Entrepreneurship, v. 7, n. 1, p. 1-24, 2016.

BENNIE, Andrew; MARLIN, Demelza; APOIFIS, Nick; WHITE, Rhiannon. 'We were made to feel comfortable and ... safe': co-creating, delivering, and evaluating coach education and health promotion workshops with Aboriginal Australian peoples. Annals of Leisure Research, 2019.

BIRMAN, Eliane. Avaliação de egressos de projetos sociais: o que sabemos sobre o pós-projeto? 2016. Dissertação (Mestrado em Engenharia de Produção) - Instituto Alberto Luiz Coimbra de Pós-Graduação e Pesquisa de Engenharia (Coppe) da Universidade Federal do Rio de Janeiro, Rio de Janeiro, 2013. 
BRANDÃO, Daniel; CRUZ, Célia; ARIDA, Anna Lívia. Métricas em Negócios de Impacto Social: Fundamentos. São Paulo: ICE/MOVE, 2014.

BREYER, Rafael Falcão. Programa Segundo Tempo: um estudo sobre os objetivos e os processos de avaliação e de construção de indicadores de impacto. 2017. Dissertação (Mestrado em Ciências do Movimento Humano) Universidade Federal do Rio Grande do Sul, Rio Grande do Sul, 2017.

CABRAL, Eloisa Helena de Souza. Valores e espaço público: referenciais e instrumentos para a avaliação de projetos sociais. Revista de Administração Pública, Rio de Janeiro, v. 45, n. 6, p. 1917-1941, nov./dez., 2011.

CARDOSO, Maria Lúcia de Macedo; COSTA, Delaine Martins. O que a perspectiva antropológica tem a dizer sobre a avaliação de projetos sociais apoiados pela cooperação internacional? Horizonte Antropológico, Porto Alegre, v. 20, n. 41, p. 117-140, 2014.

\section{CILENTO, Ivo. Gestão de projetos no terceiro setor: avaliação de} projetos sociais na economia solidária. 2019. Dissertação (Mestrado em Gestão Pública e Cooperação Internacional) Universidade Federal da Paraíba, João Pessoa, 2019.

CORTÊS-NETO, Ewerton Dantas, ALCHIERI, João Carlos; MIRANDA, Hênio Ferreira de; DANTAS-CAVALCANTI, Francisco Ivo. Elaboração de indicadores de sucesso em programas de saúde pública com foco sócio-esportivo. Revista Salud Pública, Bogotá, v. 12, n. 2, p. 208-219, abr., 2010.

CRUZ FILHO, Paulo Roberto Araújo. Avaliação e mensuração de impacto. In: ANASTÁCIO, Mari Regina; CRUZ FILHO, Paulo Roberto Araújo; MARINS, James. (Org.). Empreendedorismo social e inovação social no contexto brasileiro. Curitiba: PUCPRESS / Editora Universitária Champagnat, 2018. p. 31-50.

DEBRAY, Elizabeth; McDERMOTT, Kathryn; FRANKENBERG, Erica, BLANKENSHIP, Ann Elizabeth. Lessons from a federal grant for school diversity: Tracing a theory of change and implementation of local policies. Education Policy Analysis Archives, v. 23, n. 83, 2014.

DINIZ, Luciano Matias; VOESE, Simone Bernardes. Avaliação de programas sociais: Uma análise da relação do programa bolsa família e o desenvolvimento municipal. Revista Espacios, v. 37, n. 30, 2016.

DUGAND, Adriana Gaviria; BRANDÃO, André Augusto Pereira. A Teoria da Mudança como Ferramenta Avaliativa do Desenho dos Programas Sociais: o caso das ações estruturantes para Comunidades Quilombolas. Meta:

Avaliação, v. 9, n. 25, p.110-140, 2017. 
FABIANI, Paula; REBEHY, Sofia; CAMELO, Rafael; VICENTE, Francisco José; MOSANER, Marcelo. Avaliação de Impacto Social metodologias e reflexões. São Paulo: IDIS, 2018.

GASPARINI, Max Felipe Vianna; FURTADO, Juarez. Avaliação de Programas e Serviços Sociais no Brasil: uma análise das práticas no contexto atual. Serviço Social \& Sociedade, São Paulo, n. 117, p. 122-141, jan./mar., 2014.

GIL, Antonio Carlos. Como elaborar projetos de pesquisa. 4a. Ed. São Paulo: Atlas, 2008.

GONZÁLEZ, Carlos Villarroel; GÓMEZ, Fernando Cabrales; PRADO, María Angélica Ramírez. Metodología de evaluación de impacto aplicada al programa regional de apoyo al emprendimiento de Corfo, Chile. Interciencia, v. 44, $n$. 8, p. 461-468, 2019.

GOUVEIA, Charlete Nayana Nunes Alves. Avaliação da eficácia e efetividade do Programa de Erradicação do Trabalho Infantil a partir da perspectiva dos usuários e agentes. 2013. Tese (Doutorado em Psicologia Social) - Universidade Federal da Paraíba. Paraíba, 2013.

GUTIÉRREZ, Juan Pablo; Erika Atienzo; BERTOZZI, Stefano; McPherson Sam. Effects of the Frontiers Prevention Project in Ecuador on sexual behaviours and sexually transmitted infections amongst men who have sex with men and female sex workers: Challenges on evaluating complex interventions. Journal of Development Effectiveness, v. 14, n. 2, p. 158-177, 2013.

IMBENS, Guido Wilhelmus; LEMIEUX, Thomas. Regression discontinuity designs: A guide to practice. Journal of Econometrics, v. 142, p. 615-635, 2008.

international FinANCE CORPORATION. Our People \& Practices. London: IFC ANNUAL REPORT, 2016.

JANNUZZI, Paulo de Martino. Avaliação de programas sociais: conceitos e referenciais de quem a realiza. Est. Aval. Educ., São Paulo, v. 25, n. 58, p. 22-42, maio/ago., 2014.

JANNUZZI, Paulo de Martino. Avaliação de Programas Sociais no Brasil: Repensando práticas e metodologias das metodologias avaliativas.

Planejamento e Políticas Públicas, n. 36, jan./jul., 2011.

JREIGE, Cintia Miziara. Programa de Residência Multiprofissional em

Saúde: Um estudo sobre os efeitos na crença, satisfação e comprometimento. 2013. Dissertação (Mestrado em Psicologia Social do Trabalho e das Organizações) - Universidade de Brasília. Brasília, 2013. 
KINPARA, Daniel Ioshiteru. Abordagem Multinível na Avaliação do

Programa Bolsa Família. 2013. Tese (Doutorado em Psicologia Social, do Trabalho e das Organizações) - Universidade de Brasília, Brasília, 2013.

LELIS, Lorena Vieira Costa. Transferência condicional de renda e políticas de desenvolvimento rural no Brasil: Explorando potenciais sinergias entre Bolsa Família e o Projeto Pró-Gavião. 2016. Tese (Doutorado em Economia Aplicada) - Universidade Federal de Viçosa, Viçosa, 2016.

LILLIS, Deirdre. Systematically evaluating the effectiveness of quality assurance programmes in leading to improvements in institutional performance. Quality in Higher Education, v. 18, n. 1, p. 59-73, abr., 2012.

McINNES, Melayne Morgan; OZTURK, Orgul Demet; McDERMOTT, Suzanne, MANN, Joshua. Improved Targeting of Social Programs: An Application to a State Job Coaching Program for Adults with Intellectual Disabilities. Eastern Economic Journal, v. 42, n. 2, p. 252-269, 2016.

MENEZES, Gustavo Silva. O impacto dos programas sociais na qualidade de vida de seus participantes. 2017. Tese (Doutorado em Psicologia) Universidade Salgado de Oliveira, Rio de Janeiro, 2017.

MENEZES, Gustavo Silva; MOURÃO, Luciana. Programa Minha Casa Minha Vida sob a perspectiva da qualidade de vida. Psicoperspectivas, Valparaíso, v. 16, n. 3, p. 149-163, nov., 2017.

MONTEIRO, Cláudia do Rêgo; SILVA, Leticia Ester Cruz da., ELLIOT, Ligia Gomes. Avaliação de Impacto Social da Comunidade Vida e Paz: Um exercício de meta-avaliação. Meta: Avaliação, v. 9, n. 27, p. 503-531, set./dez., 2017.

MOREIRA, Gustavo Carvalho. Externalidades do Programa Bolsa Família sobre a violência doméstica contra a mulher no Brasil. 2014. Dissertação (Mestrado em Economia Aplicada) Universidade Federal de Viçosa, Viçosa, 2014.

MURAD, Elisa Pereira. Desenvolvimento de métricas para avaliação dos impactos relacionados às ações da Organização não Governamental Amigos de Itajubá. 2017. Dissertação (Mestrado Profissional) - Universidade Federal de Itajubá, Itajubá, 2017.

NEVES, Marília Chimendes da Silva. Fatores para avaliação de projetos sociais. 2015. Dissertação (Mestrado em Engenharia de Produção) Universidade Federal de Pernambuco, Recife, 2015.

NEWELL, Sallie.; GRAHAM, Anne. Using an Empowerment Evaluation Approach with Community-based Programs: Reflections from the Front Line. Evaluation Journal of Australasia, v. 12, n. 2, p. 15-17, 2012. 
OLSEN, Sara; GALIMIDI, Brett. Social venture technology group. Catalog of approaches to impact measurement. Assessing social impact in private ventures. Version 1.1. New York: The Rockefeller Foundation, 2008.

PINTO, Cristine. Variáveis Instrumentais. In: MENEZES FILHO, Naercio Aquino; PINTO, Cristine Campos de Xavier (Org). Avaliação Econômica de Projetos Sociais. São Paulo: Itaú Social, 2012. p. 145-166.

POSPIESZNA, Paulina. Democracy assistance and women's political empowerment in post-conflict countries. Democratization, v. 22, n. 7, p. 1250-1272, jun., 2014.

RIBEIRO, Gilberto. O que o futuro reserva para a avaliação de impacto? In: Relatório: Avanços, conquistas e orientações para o futuro 2017. Disponível em: https://aliancapeloimpacto.org.br/wpcontent/uploads/2020/02/alianca-avancos-conquistas-orientacoes-web.pdf. Acesso em: 15 out. 2020.

RIBEIRO, Marcelo Silva de Souza; RIBEIRO, Carla Valois. Saúde e Prevenção nas Escolas (SPE): elementos para avaliação de projetos sociais em Juazeiro, Bahia, Brasil. Interface, Botucatu, v. 19, n. 53, p. 337-348, mar., 2015. RICHARDSON, Roberto Jarry. Pesquisa social: métodos e técnicas. $4^{a}$. Ed., São Paulo: Atlas, 2017.

ROCHA, Mirian Aparecida. Impacto do Programa Bolsa Família sobre os padrões de consumo das famílias brasileiras. 2015. Tese (Doutorado em Economia Aplicada) - Universidade Federal de Viçosa, Viçosa, 2015.

ROCHE, Chris. Avaliação de impacto dos trabalhos de ONGs: aprendendo a valorizar as mudanças. 2a. Ed. São Paulo: Cortez, ABONG, 2000.

ROSENBAUM, Paul; RUBIN, Donald. The central role of the propensity score in observational studies for causal effects. Biometrika, v. 70, n. 1, p. 41-55, abr., 1983.

SAUMA, Enzo; VERA, Sonia; OSORIO, Karim; VALENZUELA, Deinny. Design of a methodology for impact assessment of energy efficiency programs:

measuring indirect effects in the Chilean case. Energy Efficiency, v. 9, n. 3, p. $699-721,2016$.

SERAPIONI, Mauro. Conceitos e métodos para a avaliação de programas sociais e políticas públicas. Sociologia, Porto, v. 31, p. 59-80, jun., 2016.

SILVA, Luciana Pires da. Construção e validação de instrumento de avaliação dos projetos sociais do Programa Petrobras Socioambiental. 
2015. Dissertação (Mestrado em Avaliação) - Fundação Cesgranrio, Rio de Janeiro, 2015.

SILVA, Ricardo Kleiber Lima. Avaliação da implementação do plano de mobilidade urbana em Natal - RN. 2018. Tese (Doutorado em Ciências Sociais) - Universidade Federal do Rio Grande do Norte, Rio Grande do Norte, 2018.

STERN, Elliot; STAME, Nicoletta; MAYNE, John; FORSS, Kim; DAVIES, Rick, BEFANI, Barbara. Broadening the range of designs and methods for impact evaluations. Working Paper 38. London: Department for International Development, 2012.

TAÑO, Bruna Lidia. A constituição de ações intersetoriais de atenção às crianças e adolescentes em sofrimento psíquico. 2017. Tese (Doutorado em Educação Especial) -

Universidade Federal de São Carlos, São Carlos, 2017.

UNGAR, Michael; DUQUE, Luiz; HERNANDEZ, Dora. Can focus groups be used for longitudinal evaluation? Findings from the Medellin early prevention of aggression program. International Journal of Multiple Research

Approaches, v. 5, n. 1, p. 40-51, 2014.

VASCONCELOS, Marisa de. Projeto Criança Feliz: um estudo de caso da aplicação da cosmovisão calvinista de ação social. 2014. Dissertação (Mestrado em Ciência da Religião) -

Universidade Presbiteriana Mackenzie, São Paulo, 2014.

VELDMAN, Paul. Learning Social Metrics from International

Development. Columbia University. 2009. Disponível em:

https://www.frbsf.org/community-development/files/Veldman.pdf. Acesso em:

27 out. 2019.

WHITE, Howard. An introduction to the use of randomised control trials to evaluate development interventions. Journal of Development

Effectiveness, p. 30-49, Mar. 2013. Disponível em:

https://www.tandfonline.com/doi/full/10.1080/19439342.2013.764652.

Acesso em: 27 out. 2019.

ZARYCHTA, Alan; KRISTER, Anderson; ROOT, Elisabeth; MENKEN, Jane; GRILLOS, Tara. Assessing the impacts of governance reforms on health services delivery: a quasi-experimental, multi-method, and participatory approach. Health Services and Outcomes Research Methodology, v. 19, n. 4, p. 241-258, 2019. 
Recebido em: 5 de fevereiro de 2021. Aceito em: 25 de março de 2021. Publicado em: 15 de dezembro de 2021. 